\title{
Structure and composition of C-S-H compounds up to 143 GPa
}

\author{
Elena Bykova $\odot,{ }^{1}$ Maxim Bykov $\odot,{ }^{1,2}$ Stella Chariton $\odot,{ }^{3}$ Vitali B. Prakapenka $\odot,{ }^{3}$ Konstantin Glazyrin $\odot,{ }^{4}$ \\ Andrey Aslandukov $\odot,{ }^{5}$ Alena Aslandukova $\odot,{ }^{6}$ Giacomo Criniti, ${ }^{6}$ Alexander Kurnosov, ${ }^{6}$ and Alexander F. Goncharov ${ }^{1}$ \\ ${ }^{1}$ Earth and Planets Laboratory, Carnegie Institution of Washington, 5251 Broad Branch Road NW, Washington, DC 20015, USA \\ ${ }^{2}$ Department of Mathematics, Howard University, Washington, DC 20059, USA \\ ${ }^{3}$ Center for Advanced Radiations Sources, University of Chicago, Chicago, Illinois 60637, USA \\ ${ }^{4}$ Photon Science, Deutsches Elektronen-Synchrotron, Notkestrasse 85, 22607 Hamburg, Germany \\ ${ }^{5}$ Material Physics and Technology at Extreme Conditions, Laboratory of Crystallography, \\ University of Bayreuth, 95440 Bayreuth, Germany \\ ${ }^{6}$ Bavarian Research Institute of Experimental Geochemistry and Geophysics (BGI), University of Bayreuth, \\ Universitaetsstrasse 30, 95440 Bayreuth, Germany
}

(Received 18 December 2020; revised 14 March 2021; accepted 9 April 2021; published 20 April 2021; corrected 11 May 2021)

\begin{abstract}
We synthesized two C-S-H compounds from a mixture of carbon and sulfur in hydrogen $-\mathrm{C}:\left(\mathrm{H}_{2} \mathrm{~S}\right)_{2} \mathrm{H}_{2}$ and from sulfur in mixed methane-hydrogen fluids- $\left(\mathrm{CH}_{4}\right)_{x}\left(\mathrm{H}_{2} \mathrm{~S}\right)_{(2-x)} \mathrm{H}_{2}$ at $4 \mathrm{GPa}$. X-ray synchrotron single-crystal diffraction and Raman spectroscopy have been applied to these samples up to 58 and $143 \mathrm{GPa}$, respectively. Both samples show a similar $\mathrm{Al}_{2} \mathrm{Cu}$-type $14 / \mathrm{mcm}$ basic symmetry, while the hydrogen subsystem evolves with pressure via variously ordered molecular and extended modifications. The methane-bearing sample lowers symmetry to an orthorhombic Pnma structure after laser heating to $1400 \mathrm{~K}$ at $143 \mathrm{GPa}$. The results suggest that C-S-H compounds are structurally different from a common Im-3m $\mathrm{H}_{3} \mathrm{~S}$.
\end{abstract}

DOI: 10.1103/PhysRevB.103.L140105

The concept of doped hydrogen metallic alloys [1] has been reinvigorated in the last several years fueled by developments of high-pressure experimental techniques and more advanced computational methods. Recent discoveries of high-temperature superconductivity in poly- and superhydrides [2-7] at high pressures (100-270 GPa) call for investigations of the properties related to its emergence. However, the experiments at such extreme pressures remain very challenging especially in combining different experimental techniques often making uncertain the conclusions about the nature of superconductivity (e.g., Ref. [8]). In particular, the direct structural determination including the hydrogen positions remains problematic and is commonly addressed using first-principles density-functional theoretical calculations (e.g., Ref. [9]). Also, the superconductivity probes are often not fully convincing as the direct measurements of the Meissner effect are extremely challenging [10]. Theoretical models mostly lean toward strong electron-phonon coupling mechanisms, which involve high-frequency hydrogen phonon modes [11,12], where anharmonicity plays an important role as well the hydrogen crystallographic positions and dynamics [13]. However, these theories are challenged by alternative or more complex theoretical descriptions (e.g., Refs. [14,15]). Moreover, the whole notion of high-temperature superconductivity is questioned because of the alleged inconsistencies in the superconducting probes [16-18].

Historically, sulfur polyhydride, $\mathrm{H}_{3} \mathrm{~S}$ was the first material which demonstrated a very high superconducting transition temperature $T_{c}$ of $203 \mathrm{~K}$ at $150 \mathrm{GPa}$ [2]. This material has an unusual for ambient pressure composition and can be considered as being disproportionated from a familiar molecular $\mathrm{H}_{2} \mathrm{~S}$ [19]. However, at low pressures a host-guest compound $\left(\mathrm{H}_{2} \mathrm{~S}\right)_{2} \mathrm{H}_{2}$ with the $\mathrm{Al}_{2} \mathrm{Cu}$ structure $(\mathrm{I} / \mathrm{mcm}$ symmetry) has been found, which has the same stoichiometry as $\mathrm{H}_{3} \mathrm{~S}$ high-temperature superconductor [20]. The pressuredriven transformation pathway between these two phases has been demonstrated on the pressure decrease upon temperature annealing to overcome the kinetic hindrance [21], while on the compression path $I 4 / \mathrm{mcm}$ or $\mathrm{Cccm}$ (at higher pressures) $\left(\mathrm{H}_{2} \mathrm{~S}\right)_{2} \mathrm{H}_{2}$ remains stable up to $160 \mathrm{GPa}[22,23]$. This is again due to kinetic reasons because $C c c m\left(\mathrm{H}_{2} \mathrm{~S}\right)_{2} \mathrm{H}_{2}$ and $I m-3 m$ $\mathrm{H}_{3} \mathrm{~S}$ are very different structurally, and the transition between them involves the volume discontinuity of more than $4 \%$ (at $110 \mathrm{GPa}$ ) [21]. However, $\mathrm{Im}-3 m \mathrm{H}_{3} \mathrm{~S}$ has been reported to form above $140 \mathrm{GPa}$ from the elements or reaction products of $\mathrm{H}_{2} \mathrm{~S}$ by temperature cycling below $300 \mathrm{~K}$ [24] or with the assistance of gentle laser heating [21,25] $(<1300 \mathrm{~K})$.

Recently, Snider et al. [7]. reported much higher values of $T_{c}$ in a mixed $\mathrm{C} / \mathrm{S}$ polyhydride compressed up to 267 GPa. Based on Raman spectroscopy observations, the material synthesized at $4 \mathrm{GPa}$ was suggested to be the $\left(\mathrm{CH}_{4}\right)_{x}\left(\mathrm{H}_{2} \mathrm{~S}\right)_{(2-x)} \mathrm{H}_{2}$ compound with $\mathrm{I} 4 / \mathrm{mcm}$ symmetry [20], but no direct structural determination was presented. The substitution of $\mathrm{H}_{2} \mathrm{~S}$ by methane is plausible because both molecules have similar kinetic diameters; however, the carbon composition of the material they synthesized was not determined. Moreover, since no Raman signal has been recorded at pressures above $60 \mathrm{GPa}$, the structure of the superconducting phase could not be determined or traced. Theoretical calculations on this system $[26,27]$ showed dynamic stability of a 
(a)
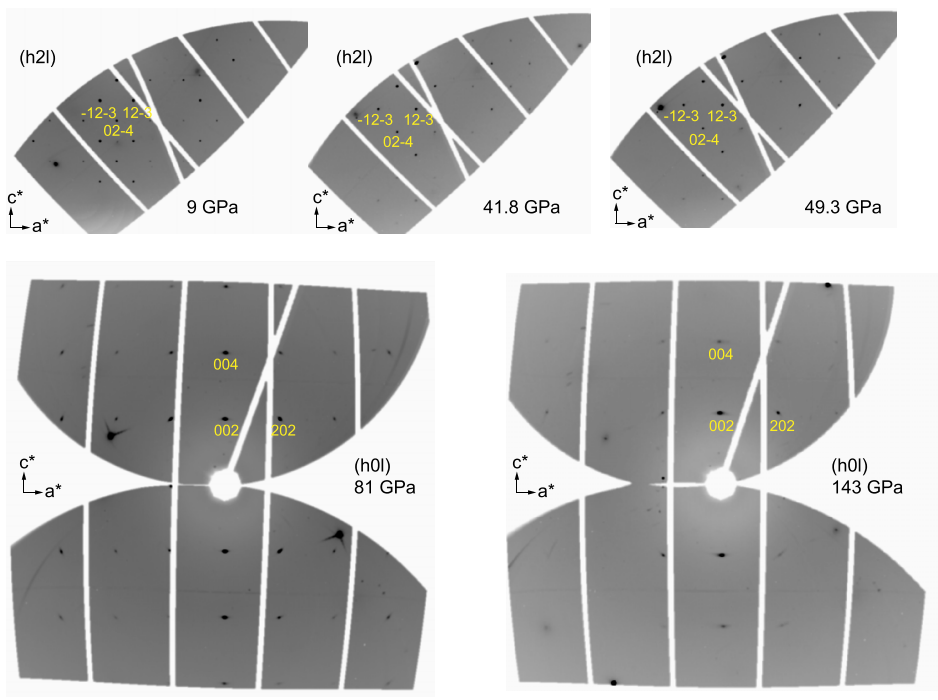

(b)

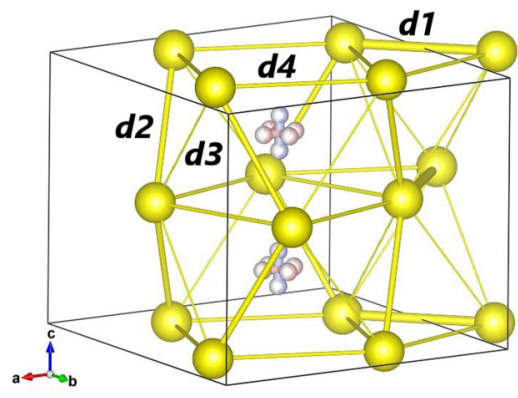

FIG. 1. XRD results on C-S-H compounds at high pressures. (a) Reconstructed reciprocal lattice plane defined by [100] and [001] directions of methane-substituted B2 batch samples at various pressures. (b) The structural model of $I 4 / m c m\left(\mathrm{H}_{2} \mathrm{~S}\right)_{2} \mathrm{H}_{2}$ (see Supplemental Material for the unit-cell parameters and atomic positions). Only the $\mathrm{H}_{2}$ molecules were included in the structural model: those oriented along the $c$ axis and in the $\boldsymbol{a} \boldsymbol{b}$ plane below and above $14 \mathrm{GPa}$ are both shown. The S-S closest distances are labeled. The distances marked as $d 1$ and $d 4$ are located in $a b$ plane, while $d 2$ and $d 3$ distances connect $\mathrm{S}(\mathrm{C})$ atoms lying on the neighboring $a b$ planes. Following laser heating at $143 \mathrm{GPa}$, we find a distortion of the unit cell, which becomes orthorhombic Pnma, but the arrangement of sulfur atoms repeats one in the parent $I 4 / m c m$ structure; the positions of hydrogen atoms could not be determined. The potential positions of hydrogen atoms forming hydrogen bonds in the $\mathrm{S}$ cages can be inferred by analyzing the $\mathrm{P}$ dependencies of the $d 1$ to $d 4$ [Fig. 2(a)].

mixed $\mathrm{CSH}_{7}$ hydride above $100 \mathrm{GPa}$, where $\mathrm{CH}_{4}$ molecules substitute $\mathrm{SH}_{6}$ units in $\mathrm{Im}-3 m \mathrm{H}_{3} \mathrm{~S}$ causing a lattice distortion. This material is found to be a high-temperature superconductor comparable to $\mathrm{H}_{3} \mathrm{~S}$ but no sharp increase of $T_{c}$ with pressure as reported by Snider et al. [7] is predicted. Here we investigated two $\mathrm{C}-\mathrm{S}-\mathrm{H}$ materials referred to hereafter as B1 and B2 (see details in Supplemental Material [28]) synthesized similarly to Ref. [7] with elementary carbon and methane used as the carbon sources, respectively, and explored their structure, vibrational and optical properties using single-crystal x-ray diffraction (SCXRD), Raman and visible absorption spectroscopy, concomitantly in a diamond anvil cell (DAC) up to $143 \mathrm{GPa}$. We determined that the structures of these alloys are very similar, and they resemble that of I4/mcm $\left(\mathrm{H}_{2} \mathrm{~S}\right)_{2} \mathrm{H}_{2}$, which slightly distorts but retains the main structural motif even after laser heating to beyond $2400 \mathrm{~K}$ at $143 \mathrm{GPa}$. This suggests that carbon stabilizes $\mathrm{Al}_{2} \mathrm{Cu}$-type structure, which may be responsible for a great increase in superconductivity at high pressures.

We examined four samples of two different kinds at various pressures by SCXRD at the GeoSoilEnviroCARS beamline (Advanced Photon Source, ANL, Chicago) and the Extreme Conditions Beamline at Petra III (DESY, Hamburg). The details of the sample preparation, data collection, structure determination, and refinement are presented in the Supplemental Material [28]. The exact determination of hydrogen positions attached to $\mathrm{S}$ atoms was not possible, due to low scattering power of hydrogen compared to sulfur and likely due to strong degree of disorder in $\mathrm{H}_{2} \mathrm{~S}$ molecules (see below). On the other hand, the location of hydrogen molecules could be determined by analyzing residual density maps. Hydrogen molecules were introduced in the structure in order to describe the highest residual density reflections; additionally, we used constraints on the $\mathrm{H}-\mathrm{H}$ distances $(0.75 \pm 0.02 \AA$ according to Duan et al. [29]); this procedure improved $R_{1}$ values by $0.5-1$ percentage points. For the B1 and B2 samples, the substitution of sulfur by carbon does not improve the refinement, since the basic $I 4 / \mathrm{mcm}$ structure has only one crystallographic position for $\mathrm{S}$ atoms, which can be randomly substituted by $\mathrm{C}$ atoms, and its refined occupancy is directly correlated with a scale factor.

The basic structure of the material is defined solely by sulfur positions and belongs to tetragonal I4/mcm $\left(\mathrm{H}_{2} \mathrm{~S}\right)_{2} \mathrm{H}_{2}$ [20] type. We find that below $14 \mathrm{GPa} \mathrm{H}_{2}$ molecules are oriented parallel to the $c$ axis and located in the cavities formed by sulfur (Fig. 1(b)). The symmetry positions of $\mathrm{S}$ atoms (Tables S2-S4 [28]) remain unchanged while the fractional coordinates change little to the highest studied pressure (143 GPa). The reciprocal-space reconstructions [Fig. 1(a)] demonstrate that neither systematic absence violations for $I$ lattice nor new reflections appear; moreover, the diffraction peaks do not show any splitting. Also, we find no orthorhombic distortion (e.g., in Cccm structure as suggested in Ref. [29]) of the basic structure up to the highest reached pressures for both B1 and B2 materials. However, in the B1 batch sample at $27 \mathrm{GPa}$ additional reflections were observed nearby the reflections belonging to the $I 4 / \mathrm{mcm}$ phase. The new phase was indexed in monoclinic $C$-centered unit cell with parameters $a=8.255(16), b=6.3944(18), c=12.14(3) \AA, \beta=$ $99.3(3)^{\circ}$ (Table S5 [28]). This corresponds to the tripling of the $I 4 / \mathrm{mcm}$ unit cell tentatively accounting for very complex Raman spectra observed in this pressure range (see below).

Under compression above $14 \mathrm{GPa}$ the refinement agreement factors are systematically better if the $\mathrm{H}_{2}$ molecules initially oriented along the $c$ axis are rotated by $90^{\circ}$ so they become parallel to the $\boldsymbol{a} \boldsymbol{b}$ plane. In the refinement 

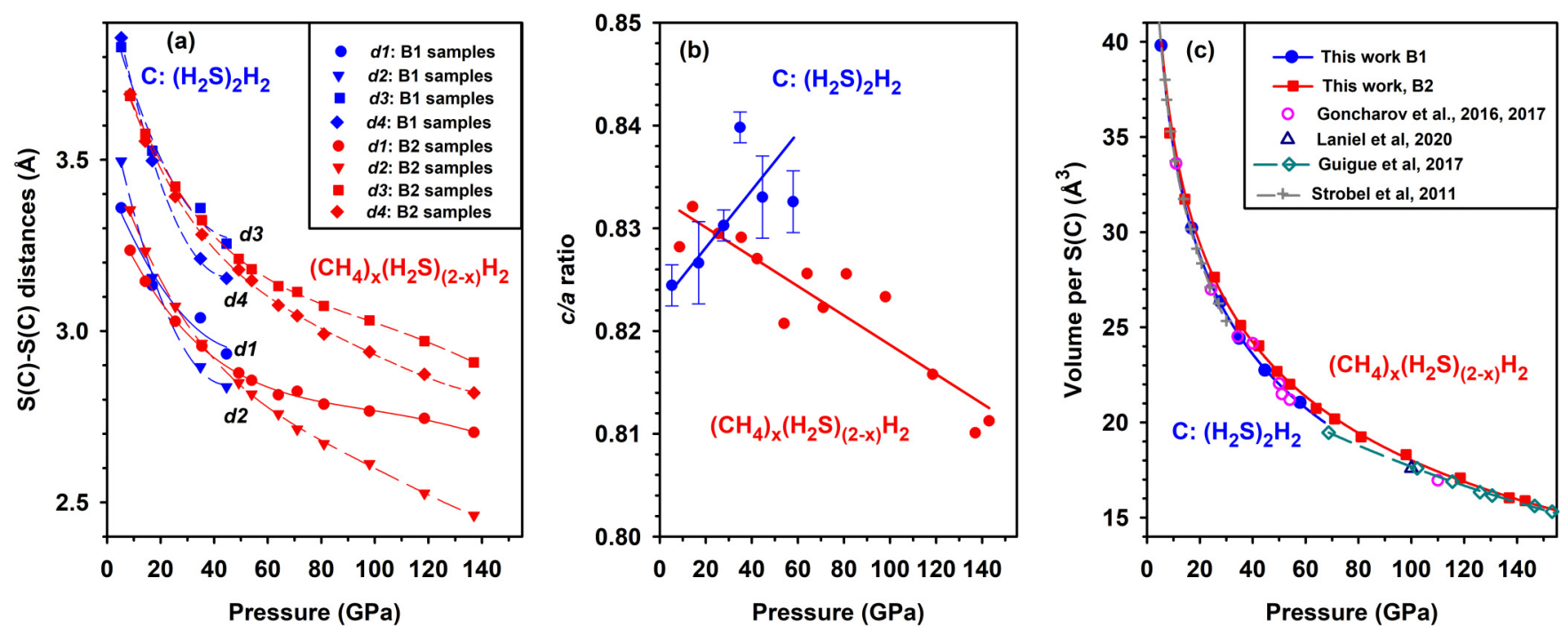

FIG. 2. The unit cell and structure parameters determined in single-crystal XRD experiments. The results for both B1 and B2 batches are shown in blue and red, respectively. (a) The closest $\mathrm{S}(\mathrm{C})-\mathrm{S}(\mathrm{C})$ distances. (b) The $\boldsymbol{c} / \boldsymbol{a}$ lattice parameters ratio. (c) The volumes per one $\mathrm{S}(\mathrm{C})$ atom; the results of this work for batches B1 and B2 (carbon and methane originated) are fit with the Vinet equation of state $\left(P_{0}=\right.$ 4.8 GPa, $\left.V_{0}=41(1) \AA^{3}, K_{0}=23(2) \mathrm{GPa}, K_{0}^{\prime}=4.3(3)\right)$. The error bars are smaller than the symbol size. Our data are compared to those of Refs. [19-22,30] for Cccm $(I 4 / \mathrm{mcm})\left(\mathrm{H}_{2} \mathrm{~S}\right)_{2} \mathrm{H}_{2}$; the results of Pace et al. [23], which agree well with other data at high pressures but scatter and largely disagree below $60 \mathrm{GPa}$, are not shown for clarity.

such molecules were approximated by two perpendicular molecules with occupancy factor of 0.5 to keep the fourfold axis [Fig. 1(b)]. No further changes in the orientation of $\mathrm{H}_{2}$ molecules were detected up to $143 \mathrm{GPa}$.

While the location of $\mathrm{H}$ atoms in $\mathrm{S}(\mathrm{C})$ cages was not possible, the shortest $\mathrm{S}(\mathrm{C})-\mathrm{S}(\mathrm{C})$ distances deduced from our single-crystal XRD data [Fig. 2(a)] could be used to infer their positions. The $\mathrm{S}(\mathrm{C})-\mathrm{S}(\mathrm{C})$ distances in the samples of both types contract similarly under pressure swapping the ranking between $d 1$ and $d 2$ and splitting $d 3$ and $d 4$. At about $50 \mathrm{GPa}$, where the Raman spectra dramatically change signaling the formation of an extended solid (see below), the $d 3$ and $d 4$ distances are in the range of 3.13-3.2 $\AA$, corresponding to a regime of the strong hydrogen bonding near symmetrization [29]. The $d 1$ and $d 2$ distances are shorter than one would accept if there were a $\mathrm{H}$ atom between them forming a nearly linear hydrogen bond. These results suggest that $\mathrm{H}$ atoms in the $\mathrm{S}(\mathrm{C})$ cages are likely positioned along the lines corresponding to $d 3$ and $d 4 \mathrm{~S}(\mathrm{C})-\mathrm{S}(\mathrm{C})$ distances.

There is a difference in the behavior of samples from batches B1 and B2 which can be assigned to a different kind of substitution of $\mathrm{S}$ by $\mathrm{C}$. In the samples of the batch B1, which do not contain the methane as a building block, carbon (if any) likely substitutes $\mathrm{H}_{2} \mathrm{~S}$ molecules (thereby creating vacancies)-we call it C-doped $\left(\mathrm{H}_{2} \mathrm{~S}\right)_{2} \mathrm{H}_{2}$. In the samples of batch $\mathrm{B} 2$, which do contain methane, it supposedly substitutes $\mathrm{H}_{2} \mathrm{~S}$ molecule forming $\left(\mathrm{CH}_{4}\right)_{x}\left(\mathrm{H}_{2} \mathrm{~S}\right)_{(2-x)} \mathrm{H}_{2}$. It is interesting that $\mathrm{B} 1$ and $\mathrm{B} 2$ samples demonstrate a distinct compression anisotropy [Fig. 2(b)]: the methane-containing samples are more compressible along the $\boldsymbol{c}$ axis, while carbondoped samples are more compressible in the $\boldsymbol{a}-\boldsymbol{b}$ plane. At nearly $4 \mathrm{GPa}$, where they were synthesized, the samples have indistinguishable lattice parameters. Under pressure, a systematic difference in volumes for batches B1 and B2 develops.
The samples synthesized from $\mathrm{S}: \mathrm{CH}_{4}: \mathrm{H}_{2}$ mixtures have larger volumes, while those synthesized from $\mathrm{C}: \mathrm{S}: \mathrm{H}_{2}$ have the equation of state (EOS), which is very close to that of $\left(\mathrm{H}_{2} \mathrm{~S}\right)_{2} \mathrm{H}_{2}$ synthesized by various techniques at diverse $P$ $T$ conditions [Fig. 2(c)]. The B1 material synthesized here seems to have slightly larger volume in the limit of high pressures, but the difference is within the experimental uncertainties. It is interesting that the EOS of $\mathrm{B} 2$ samples merges with that of pure $\left(\mathrm{H}_{2} \mathrm{~S}\right)_{2} \mathrm{H}_{2}$ at high pressures. While the experimental EOS of $\left(\mathrm{H}_{2} \mathrm{~S}\right)_{2} \mathrm{H}_{2}$ has been shown to be in a good agreement with theoretical calculations, no theoretical results has been reported for mixed $\left(\mathrm{CH}_{4}\right)_{x}\left(\mathrm{H}_{2} \mathrm{~S}\right)_{(2-x)} \mathrm{H}_{2}$ compounds with $I 4 / \mathrm{mcm}$ or $\mathrm{Cccm}$ structure.

At the highest reached pressure of $143 \mathrm{GPa}$, the B2 sample was laser heated twice (both at GSECARS and ECB at Petra-III) up to a maximum temperature of $2700 \mathrm{~K}$. Already after the first heating the sample recrystallized in a multigrain phase. The grain with the most intense Bragg spots was indexed in a primitive $(P)$ orthorhombic unit cell with parameters $a=7.591(5), b=4.439(13), \quad c=7.641(6) \AA$ (Table S6 [28], Fig. S2). The unit-cell volume is doubled compared to the parent $I 4 / \mathrm{mcm}$ structure. Indeed, the reciprocal-space reconstructions show appearance of the reflections violating $I$-lattice symmetry and additional superlattice reflections at $0.5\left(\boldsymbol{a}^{*}+\boldsymbol{b}^{*}\right)$. The best structure solution has been achieved within space group Pnma, where only positions of $S$ atoms can be located. The interatomic S ...S distances vary widely from to 2.401(7) to 3.004(6) $\AA$, but general arrangement of the heavy atoms still repeats one of the $14 / \mathrm{mcm}$ structure. Such distances would also suggest strong hydrogen bonding in the structure. The increase in the unit-cell volume by $1.7(2) \AA^{3}$ compared to $I 4 / \mathrm{mcm}$ volume before heating suggests that this distorted structure could have incorporated one extra $\mathrm{H}$ atom (e.g., Ref. [31]) yielding $\left(\mathrm{CH}_{4}\right)_{x}\left(\mathrm{H}_{2+\delta} \mathrm{S}\right)_{(2-x)} \mathrm{H}_{2}$, where $\delta$ can vary from 0.125 to 0.25 if $0<x<1$. 

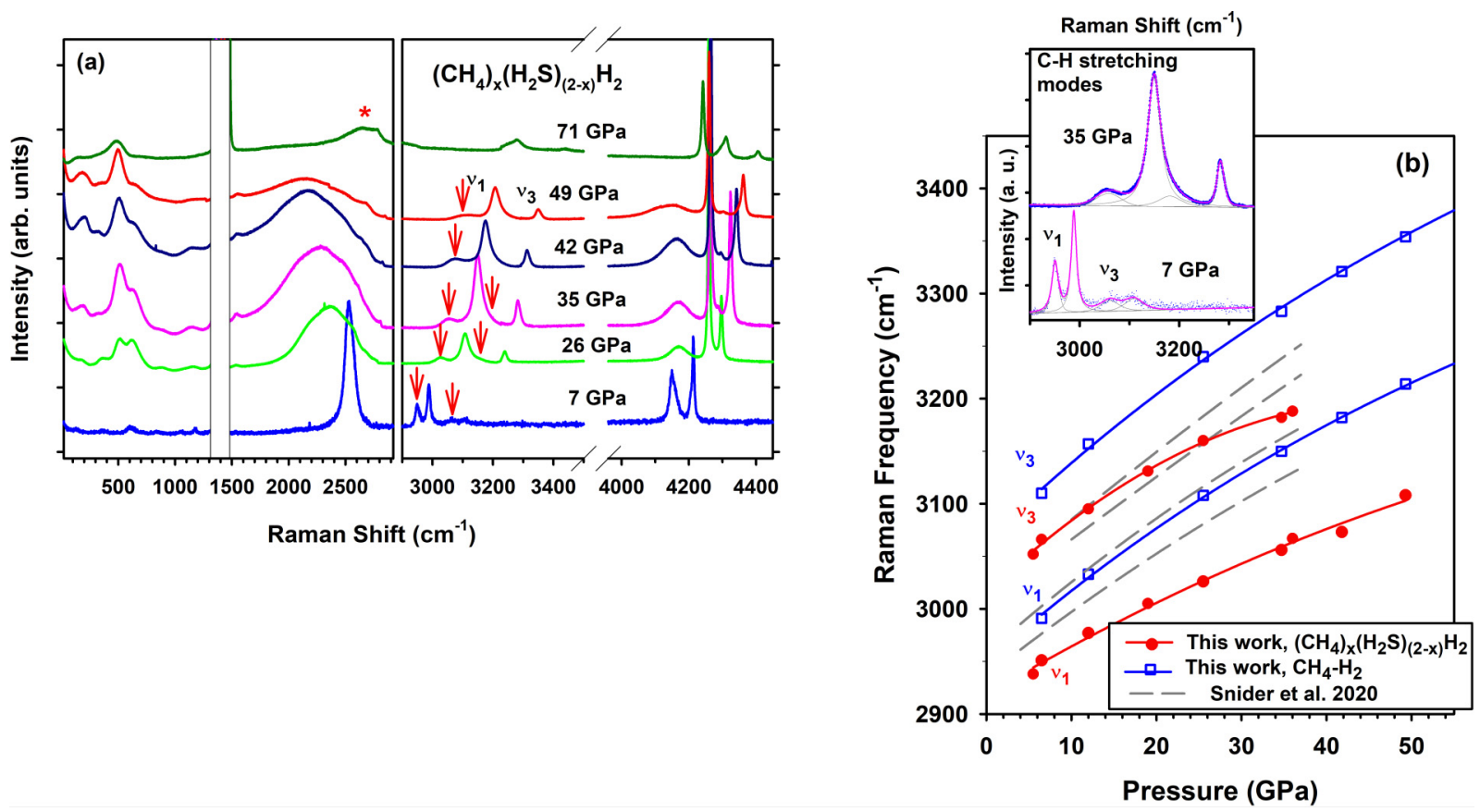

FIG. 3. (a) Raman spectra of the B2 batch samples as a function of pressure. Red arrows mark the C-H stretching band peaks of methane molecules, which are redshifted with respect to those of $\mathrm{a} \mathrm{CH}_{4}-\mathrm{H}_{2}$ compound surrounding them. The first-order diamond signal of diamond anvils is masked by a rectangle, while the second-order diamond anvil signal at $71 \mathrm{GPa}$ is marked by an asterisk. (b) Pressure dependence of the C-H Raman modes measured here on the B2 samples in comparison with the results of Snider et al. [7]. The inset shows an enlarged view of the Raman C-H stretch modes, where the data (dots) are fit using a phenomenological model (solid pink lines) and the results of the peak deconvolution are shown (gray lines).

To learn more about the $\mathrm{H}$-atom positions and $\mathrm{C}$ composition of our samples, we concomitantly investigated Raman spectra of the samples probed by XRD and other samples, which were synthesized similarly. The Raman spectra of B1 samples synthesized in this work (Fig. S3) are very similar to those reported in Ref. [7], except no sharp C-H modes have been detected. They are also similar to Raman spectra of $\left(\mathrm{H}_{2} \mathrm{~S}\right)_{2} \mathrm{H}_{2}$ reported previously [20,21,23]. This includes the behavior of the lattice, S-H bending, and stretching, and H-H stretching modes. Similarly to reported in Ref. [7] for the proposed methane-bearing material, these data show a sequence of phase transitions at approximately 15, 35, and $45 \mathrm{GPa}$ manifesting two-stage ordering of $\mathrm{H}_{2} \mathrm{~S}$ molecules and their polymerization via formation of symmetric hydrogen bonds $[20,21,23]$. Our B1 samples show a similar transformation sequence (Fig. S3) suggesting that they contain little if any carbon with an unknown substitution pattern. This is consistent with our structural data and volume vs pressure dependencies (Figs. 1 and 2). The Raman spectra above 25 GPa show very complex structure for all types of vibrations. This is consistent with the XRD observations of the superstructure, suggesting that the observed mode splitting is due to the Brillouin-zone folding, which makes the zone boundary vibrations Raman active (e.g., Ref. [32]). Above 45 GPa, the majority of the Raman bands disappear, and only weak, broad low-frequency bands remain that can be assigned to S-S modes similar to observations in $\mathrm{H}_{2} \mathrm{~S}$ [19].

In contrast, our B2 samples do show the incorporation of methane molecules into the same lattice manifested by the appearance of redshifted C-H stretching $v_{1}$ and $v_{3}$ modes
(Fig. 3), which can be distinguished from methane molecules incorporated in an inclusion compound with molecular $\mathrm{H}_{2}$ [33]. The major features of the pressure behavior of $\mathrm{B} 2 \mathrm{ma}-$ terials are similar to those for $\mathrm{B} 1$ materials but there are important differences. The lattice mode spectrum is much simpler, showing just a few broad bands: the S-H and $\mathrm{H}-\mathrm{H}$ stretching modes have less components; their softening behavior is much less pronounced including the frequency shift and pressure-induced peak broadening. Instead, the associated peaks are broadened at the transition to a state, where $\mathrm{H}_{2} \mathrm{~S}$ molecules are expected to cease rotations (above $15 \mathrm{GPa}$ ), and likely form static orientationally disordered state. As in the case of B1 samples, the Raman spectra of methane-bearing B2 samples change drastically above $50 \mathrm{GPa}$ with all the major peak vanishing and only a few lattice modes preserving higher pressures. This indicates the transformation of this material into an extended state.

The optical properties of both materials synthesized here change under pressure. While they are translucent at low pressure (Fig. S1), they gradually become dark and transmit little in extended high-pressures states. The absorption spectra of B1 C-doped $\left(\mathrm{H}_{2} \mathrm{~S}\right)_{2} \mathrm{H}_{2}$ samples show a broad absorption edge, which shifts to lower energies at high pressures, but the sample remained a narrow-band semiconductor up to the highest pressure reached of $58 \mathrm{GPa}$ (Fig. 4). In contrast, B2 samples in an extended state at $58 \mathrm{GPa}$ show a featureless absorption suggesting indirect gap closure or semimetal behavior. It is interesting that the sample of the same batch measured after the laser heating at $143 \mathrm{GPa}$ opens a band gap, likely due to hydrogen ordering. 

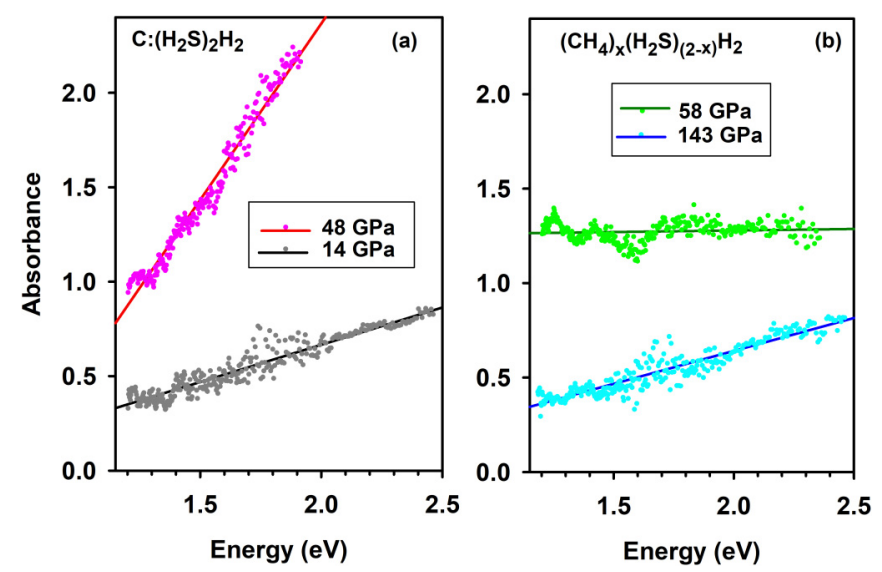

FIG. 4. Optical absorption spectra at various pressure of (a) B1 C-doped $\left(\mathrm{H}_{2} \mathrm{~S}\right)_{2} \mathrm{H}_{2}$ and (b) B2 $\left(\mathrm{CH}_{4}\right)_{x}\left(\mathrm{H}_{2} \mathrm{~S}\right)_{(2-x)} \mathrm{H}_{2}$. Dots are the data and lines are guides to the eye. The $\mathrm{B} 2$ sample at $143 \mathrm{GPa}$ is probed after the laser heating experiment. The spectra are normalized to the transmission of the medium $-\mathrm{H}_{2}$ and mixed $\mathrm{H}_{2}-\mathrm{CH}_{4}$ medium, respectively.

Our combined XRD, Raman, and optical spectroscopy investigation up to $143 \mathrm{GPa}$ provides previously missing information about the composition, and pressure-dependent structure and electronic properties of the reported roomtemperature superconducting C-S-H compounds. The composition and thus properties strongly depend on the synthesis conditions and procedure. Our extensive attempts to reproduce synthesis of $\left(\mathrm{CH}_{4}\right)_{x}\left(\mathrm{H}_{2} \mathrm{~S}\right)_{(2-x)} \mathrm{H}_{2}$ compounds from a mixture of $\mathrm{C}$ and $\mathrm{S}$ in fluid $\mathrm{H}_{2}$ medium [7] were unsuccessful in that the materials we obtained did not show any sign of $\mathrm{C}-\mathrm{H}$ bonds (Fig. S3). We argue that the materials reported by Snider et al. [7] did not incorporate as much methane molecules as they infer $(x=0.5)$, thus making problematic a direct comparison with theoretical predictions $[26,27]$. Indeed, our B2 samples synthesized in a mixed $\mathrm{H}_{2}$ and $\mathrm{CH}_{4}$ gas do show such incorporation beyond any doubt, as both XRD and Raman provide clear evidence for such mixed crystal (Figs. 1-3, Fig. S2). However, these samples show clearly different Raman spectra compared to those reported in Ref. [7] [Fig. 3(a)]. Moreover the comparison of the pressure dependencies of the Raman frequencies of the $\mathrm{C}-\mathrm{H}$ stretching modes [Fig. 3(b)] shows the difference between our data and those of Snider et al. [7], suggesting that their C-H bands do not belong to material of interest. We speculate that they originate from the $\mathrm{CH}_{4}-\mathrm{H}_{2}$ compounds [33] that have been synthesized in the cavity during the "photochemistry" processes.
Overall, it is clear that both our studied materials B1 and B2 were somewhat different from those reported by Snider et al. [7], thus suggesting that more work is needed to assess the connection between the structure and high-temperature superconductivity. The here revealed extended stability of $I 4 / \mathrm{mcm}$ structure of $\mathrm{CH}_{4}$-doped $\left(\mathrm{H}_{2} \mathrm{~S}\right)_{2} \mathrm{H}_{2}$ compounds underscores the importance of a future structural study of the sample with very high $T_{c}$.

In conclusion, using synchrotron single-crystal XRD technique we determined the basic symmetry of C-S-H compounds synthesized in the DAC in the conditions that are similar to those reported recently for room-temperature superconductor above $140 \mathrm{GPa}$, even though we were not able to reproduce the reported synthesis of $\left(\mathrm{CH}_{4}\right)_{x}\left(\mathrm{H}_{2} \mathrm{~S}\right)_{(2-x)} \mathrm{H}_{2}$ from a mixture of $\mathrm{S}$ and $\mathrm{C}$ in $\mathrm{H}_{2}$ medium. Instead, we find that such compounds can be synthesized by reacting $\mathrm{S}$ with a mixed $\mathrm{CH}_{4}$ and $\mathrm{H}_{2}$ gas medium. The sulfur lattice forms an $\mathrm{Al}_{2} \mathrm{Cu}$ structure of $I 4 / \mathrm{mcm}$ symmetry reduced to an orthorhombic Pnma structure after laser heating to $1400 \mathrm{~K}$ at $143 \mathrm{GPa}$ after the temperature annealing. The superstructure and lattice distortion detected at $27 \mathrm{GPa}$ signals the formation of a hydrogen ordered state. Raman spectra show a series of molecular ordering transformations in the hydrogen subsystem, which eventually results in formation of extended narrow-gap semiconducting or semimetallic solids above 60 GPa. The stability of the basic sulfur-based cage structure up to $143 \mathrm{GPa}$ even after the temperature annealing suggests considering a possibility that it may be a host of roomtemperature superconducting state (cf. Im- $3 m \mathrm{H}_{3} \mathrm{~S}$ revealing superconductivity at $203 \mathrm{~K}$ at $150 \mathrm{GPa}$ ), even though the materials investigated here were different from those where superconductivity was reported.

Parts of this research were carried out at the GeoSoilEnviroCARS (The University of Chicago, Sector 13), Advanced Photon Source (Argonne National Laboratory). GeoSoilEnviroCARS is supported by the National Science Foundation Earth Sciences (Grant No. EAR - 1634415). The Advanced Photon Source is a U.S. Department of Energy (DOE) Office of Science User Facility operated for the DOE Office of Science by Argonne National Laboratory under Contract No. DE-AC02-06CH11357. Use of the GSECARS Raman System was supported by the NSF MRI Proposal (EAR-1531583). Parts of this research were carried out at the Extreme Conditions Beamline (P02.2) at DESY, a member of Helmholtz Association. We acknowledge support by the Army Research Office accomplished under the Cooperative Agreement No. W911NF-19-2-0172 and Carnegie Institution of Washington.
[1] N. W. Ashcroft, Phys. Rev. Lett. 92, 187002 (2004).

[2] A. P. Drozdov, M. I. Eremets, I. A. Troyan, V. Ksenofontov, and S. I. Shylin, Nature (London) 525, 73 (2015).

[3] A. P. Drozdov, P. P. Kong, V. S. Minkov, S. P. Besedin, M. A. Kuzovnikov, S. Mozaffari, L. Balicas, F. F. Balakirev, D. E. Graf, V. B. Prakapenka, E. Greenberg, D. A. Knyazev, M. Tkacz, and M. I. Eremets, Nature (London) 569, 528 (2019).
[4] M. Somayazulu, M. Ahart, A. K. Mishra, Z. M. Geballe, M. Baldini, Y. Meng, V. V. Struzhkin, and R. J. Hemley, Phys. Rev. Lett. 122, 027001 (2019).

[5] P. P. Kong, V. S. Minkov, S. P. Besedin, M.A.Kuzovnikov, A. P. Drozdov, S. Mozaffari, L. Balicas, F. F. Balakirev, V. B. Prakapenka, E. Greenberg, D. A. Knyazev, and M. I. Eremets, arXiv:1909.10482 (2019). 
[6] D. V. Semenok, A. G. Kvashnin, A. G. Ivanova, V. Svitlyk, V. Y. Fominski, A. V. Sadakov, O. A. Sobolevskiy, V. M. Pudalov, I. A. Troyan, and A. R. Oganov, Mater. Today 33, 36 (2020).

[7] E. Snider, N. Dasenbrock-Gammon, R. McBride, M. Debessai, H. Vindana, K. Vencatasamy, K. V. Lawler, A. Salamat, and R. P. Dias, Nature (London) 586, 373 (2020).

[8] A. Majumdar, J. S. Tse, and Y. Yao, Angew. Chem. Int. Ed. 56, 11390 (2017).

[9] C. M. Pépin, G. Geneste, A. Dewaele, M. Mezouar, and P. Loubeyre, Science 357, 382 (2017).

[10] X. Huang, X. Wang, D. Duan, B. Sundqvist, X. Li, Y. Huang, H. Yu, F. Li, Q. Zhou, B. Liu, and T. Cui, Natl. Sci. Rev. 6, 713 (2019).

[11] K. Tanaka, J. S. Tse, and H. Liu, Phys. Rev. B 96, 100502(R) (2017).

[12] I. Errea, M. Calandra, C. J. Pickard, J. Nelson, R. J. Needs, Y. Li, H. Liu, Y. Zhang, Y. Ma, and F. Mauri, Phys. Rev. Lett. 114, 157004 (2015).

[13] I. Errea, M. Calandra, C. J. Pickard, J. R. Nelson, R. J. Needs, Y. Li, H. Liu, Y. Zhang, Y. Ma, and F. Mauri, Nature (London) 532, 81 (2016).

[14] A. Bianconi and T. Jarlborg, Novel Supercond. Mater. 1, 37 (2015).

[15] J. E. Hirsch and F. Marsiglio, Physica C (Amsterdam, Neth.) 511, 45 (2015).

[16] J. E. Hirsch and F. Marsiglio, arXiv:2010.10307v1 (2020).

[17] M. Dogan and M. L. Cohen, arXiv:2012.10771v2 (2020).

[18] E. F. Talantsev, Supercond. Sci. Technol. 34, 034001 (2021).

[19] A. F. Goncharov, S. S. Lobanov, I. Kruglov, X.-M. Zhao, X.-J. Chen, A. R. Oganov, Z. Konôpková, and V. B. Prakapenka, Phys. Rev. B 93, 174105 (2016).

[20] T. A. Strobel, P. Ganesh, M. Somayazulu, P. R. C. Kent, and R. J. Hemley, Phys. Rev. Lett. 107, 255503 (2011).

[21] A. F. Goncharov, S. S. Lobanov, V. B. Prakapenka, and E. Greenberg, Phys. Rev. B 95, 140101(R) (2017).

[22] B. Guigue, A. Marizy, and P. Loubeyre, Phys. Rev. B 95, 020104(R) (2017).

[23] E. J. Pace, X.-D. Liu, P. Dalladay-Simpson, J. Binns, M. PeñaAlvarez, J. P. Attfield, R. T. Howie, and E. Gregoryanz, Phys. Rev. B 101, 174511 (2020).

[24] M. Einaga, M. Sakata, T. Ishikawa, K. Shimizu, M. I. Eremets, A. P. Drozdov, I. A. Troyan, N. Hirao, and Y. Ohishi, Nat. Phys. 12, 835 (2016).
[25] H. Nakao, M. Einaga, M. Sakata, M. Kitagaki, K. Shimizu, S. Kawaguchi, N. Hirao, and Y. Ohishi, J. Phys. Soc. Jpn. 88, 123701 (2019).

[26] W. Cui, T. Bi, J. Shi, Y. Li, H. Liu, E. Zurek, and R. J. Hemley, Phys. Rev. B 101, 134504 (2020).

[27] Y. Sun, Y. Tian, B. Jiang, X. Li, H. Li, T. Iitaka, X. Zhong, and Y. Xie, Phys. Rev. B 101, 174102 (2020).

[28] See Supplemental Material at http://link.aps.org/supplemental/ 10.1103/PhysRevB.103.L140105 for details on materials and methods, Figs. S1-S4, and bibliography, which includes Refs. [34-41].

[29] D. Duan, Y. Liu, F. Tian, D. Li, X. Huang, Z. Zhao, H. Yu, B. Liu, W. Tian, and T. Cui, Sci. Rep. 4, 6968 (2014).

[30] D. Laniel, B. Winkler, E. Bykova, T. Fedotenko, S. Chariton, V. Milman, M. Bykov, V. Prakapenka, L. Dubrovinsky, and N. Dubrovinskaia, Phys. Rev. B 102, 134109 (2020).

[31] N. P. Salke, M. M. Davari Esfahani, Y. Zhang, I. A. Kruglov, J. Zhou, Y. Wang, E. Greenberg, V. B. Prakapenka, J. Liu, A. R. Oganov, and J.-F. Lin, Nat. Commun. 10, 4453 (2019).

[32] A. F. Goncharov, J. H. Eggert, I. I. Mazin, R. J. Hemley, and H.-k. Mao, Phys. Rev. B 54, R15590 (1996).

[33] M. S. Somayazulu, L. W. Finger, R. J. Hemley, and H. K. Mao, Science 271, 1400 (1996).

[34] V. B. Prakapenka, A. Kubo, A. Kuznetsov, A. Laskin, O. Shkurikhin, P. Dera, M. L. Rivers, and S. R. Sutton, High Pressure Res. 28, 225 (2008).

[35] C. Prescher and V. B. Prakapenka, High Pressure Res. 35, 223 (2015).

[36] CrysAlisPro Software System (Rigaku Oxford Diffraction) (Oxford, Oxford, UK, 2014).

[37] G. Sheldrick, Acta Crystallogr., Sect. A 71, 3 (2015).

[38] G. Sheldrick, Acta Crystallogr. Sect. C 71, 3 (2015).

[39] O. V. Dolomanov, L. J. Bourhis, R. J. Gildea, J. A. K. Howard, and H. Puschmann, J. Appl. Crystallogr. 42, 339 (2009).

[40] N. Holtgrewe, E. Greenberg, C. Prescher, V. B. Prakapenka, and A. F. Goncharov, High Pressure Res. 39, 457 (2019).

[41] A. F. Goncharov, P. Beck, V. V. Struzhkin, R. J. Hemley, and J. C. Crowhurst, J. Phys. Chem. Solids 69, 2217 (2008).

Correction: The affiliation for the fourth author was incorrect and has been fixed. 